\title{
Prostatic fiducial markers implantation by transrectal ultrasound for adaptive image guided radiotherapy in localized cancer: 7-years experience
}

\author{
Vito Lacetera ${ }^{1}$, Massimo Cardinali ${ }^{2}$, Giovanna Mantello ${ }^{2}$, Francesco Fenu ${ }^{2}$, Giulia Sbrollini ${ }^{1}$, \\ Flavia Tombolini ${ }^{1}$, Alessandro Conti ${ }^{1}$, Stefania Maggi ${ }^{3}$, Giovanni Muzzonigro ${ }^{1}$, Andrea Benedetto Galosi ${ }^{4}$ \\ ${ }^{1}$ Clinica Urologica, ${ }^{2}$ SOD Radioterapia, ${ }^{3}$ SOD Fisica Sanitaria Azienda Ospedaliero-Universitaria Ospedali Riuniti, \\ Università Politecnica delle Marche, Ancona; ${ }^{4}$ UOC di Urologia, Ospedale "Augusto Murri", ASUR Marche, Fermo, Italy.
}

\begin{abstract}
Summary Objective: we present our 7-years' experience with fiducial gold markers inserted before Image-Guided Radiotherapy (IGRT) focusing on our echo-guided technique reporting early and late complications.

Material and methods: 78 prostate cancer (PCA) patients who underwent fiducial markers placement for adaptive IGRT (period 2007-2014) were selected. Mean patient age was 75 years (range 60-81), mean PSA $7.8 \mathrm{ng} / \mathrm{ml}$ (range 3.1-10), clinical stage < T3, mean Gleason Score 6.4 (range 6-7). We recorded early and late complications. Maximum distance between the Clinical Target Volume (CTV) and Planning Target Volume (PTV) was assessed for each direction and the mean PTV reduction was estimated.

Results: we describe in details our echo-guided technique of intraprostatic gold fiducial markers insertion prior to adaptative IGRT. We report rare early toxicity (5-7\% grade 1-2), a mean PTV reduction of $37 \%$ and a very low late toxicity (only 3.4\% bladder G3 and 8\% rectal G2 side effects).

Conclusion: Our technique of fiducial gold markers implantation for adaptative IGRT is safe and well-tolerated and it resulted helpful to reduce CTV-PTV margin in all cases; the effects on clinical practice seem significant in terms of late toxicity but further investigations are needed with longer follow-up.
\end{abstract}

KEY WORDS: Prostate cancer; Fiducial markers; Image guided radiotherapy; Transrectal ultrasound; $C B C T$ : Cone Beam Computer Tomography; US: ultrasound; TRUS: Transrectal Ultrasound.

Submitted 3 October 2014; Accepted 31 October 2014

\section{INTRODUCTION}

Over the past few decades, advances have been made in Image-Guided Radiotherapy (IGRT) to increase the radiation dose to the prostate, while limiting toxicities to adjacent organs such as the bladder and rectum: placement of fiducial markers is an important option in IGRT treatment planning to deliver higher total doses with increased accuracy, safety, and efficacy (1). The prostate gland can be displaced by more than $1 \mathrm{~cm}$ on day-to-day radiotherapy session resulting in geographical miss of the target and unintentional irradiation of surrounding critical structures (2). For this reason, a standard margin of $1 \mathrm{~cm}$ (CTV-PTV margin) is added to Clinical Target Volume (CTV) to define the Planning Target Volume (PTV) (3). Aims of our study are to describe technique and our results of ultrasound-guided implantation of fiducial gold markers in the prostate for adaptive IGRT in lowintermediate risk cancer. Furthermore we report the measurement of CTV to define the PTV margins.

\section{MATERIALS AND METHOdS}

Seventy-eight consecutive patients who underwent fiducial markers placement using transrectal ultrasound guided technique for adaptive IGRT between January 2007 and March 2014 were analyzed. All cases were low-intermediate risk prostate cancer according to D'Amico risk classification: mean PSA was $7.8 \mathrm{ng} / \mathrm{ml}$ (range 3.1-10); clinical stage T1c-T2b based on clinical staging and rectal coil Magnetic Resonance 1.5 Tesla; biopsy Gleason Score 6.4 (range 6-7). Exclusion criteria were: high risk prostate cancer according to D'Amico risk classification or cT3. We recorded early complications of the procedure instructing patients to contact the radiation oncologists if there were any problems during the week after implantation. The Planning CT (CTsim) was acquired 7 days after implantation when markers stability was achieved. In the first 54 patients the CTV was delineated as the prostate only and a standard margin $(10 \mathrm{~mm})$ was added to define the PTV. A daily markers match between CBCT and CTsim was performed during the whole treatment, in order to correct inter-fraction prostate motion. For each patient, the first five CBCTs were then used to create a patient personalized PTV obtained as a merged volume including the 5 days CTV position (3). With the aim to calculate the gain of using gold markers, the maximum distance between the CTV and the PTV, for each direction, was assessed for each patient. The median value of the measured distances, among the whole population, per each direction, was 
indeed used to define an anisotropic margin and to compare it with the standard ones. All the patients were submitted an active follow-up. The late toxicity was recorded and classified according to the LENT SOMA score.

\section{Ultrasound technique}

Fiducial markers were inserted on ultrasound guidance with transrectal approach by the 2 referring urologist (ABG, VL) using a logic-Q P5 machine (GE Healthcare, Little Chalfont, United Kingdom) equipped with end-fire probe $(6-8 \mathrm{MHz})$ and disposable kit also used for biopsy. All patients signed an information consent describing the risks of the procedure and the rationale for undergoing fiducial marker implantation. They self-administered an enema the morning of the procedure and were placed on antibiotics prophylaxis (fluorquinolones) from the day before to 4 days after procedure. Patients were instructed to stop anticoagulant and antiplatelet medications 7 days before the implant if appropriate. Any patient, who had severe infection after prostate biopsy, was excluded from this evaluation and treated with transperineal approach. Patients with multiple and bilateral calcifications of the gland were excluded from the study and treated with IGRT since fiducial markers were considered not necessary to image the prostate with CT scan. The equipment needed and the technique are similar to that typically used during a TRUS-guided prostate biopsy: with the patient in left lateral decubitus position we instilled a topical anesthesia (2\% lidocaine-prilocain gel) and a $5 \mathrm{ml}$ povidone-iodium enema in the rectum 5 minutes before insertion of the probe. Then a local nerve block was made by injecting lidocaine 1\% (using 2lguage needle $25 \mathrm{~cm}$ long) at the angle between the seminal vesicle and prostate on either side immediate prior to insertion of the fiducials. Three fiducial markers $(0.9 \mathrm{~mm} \times 3 \mathrm{~mm})$ were placed in the prostate (lateral mid left gland, right apex and base) under ultrasound guidance. The needle was gently advanced for 1.5 centimeter into prostatic tissue and then retracted releasing

Figure 1.

A: patient position, B:TRUS guided fiducial marker's insertion (right base), C: gold markers needles, D: fluoroscopic control of fiducial markers positions.

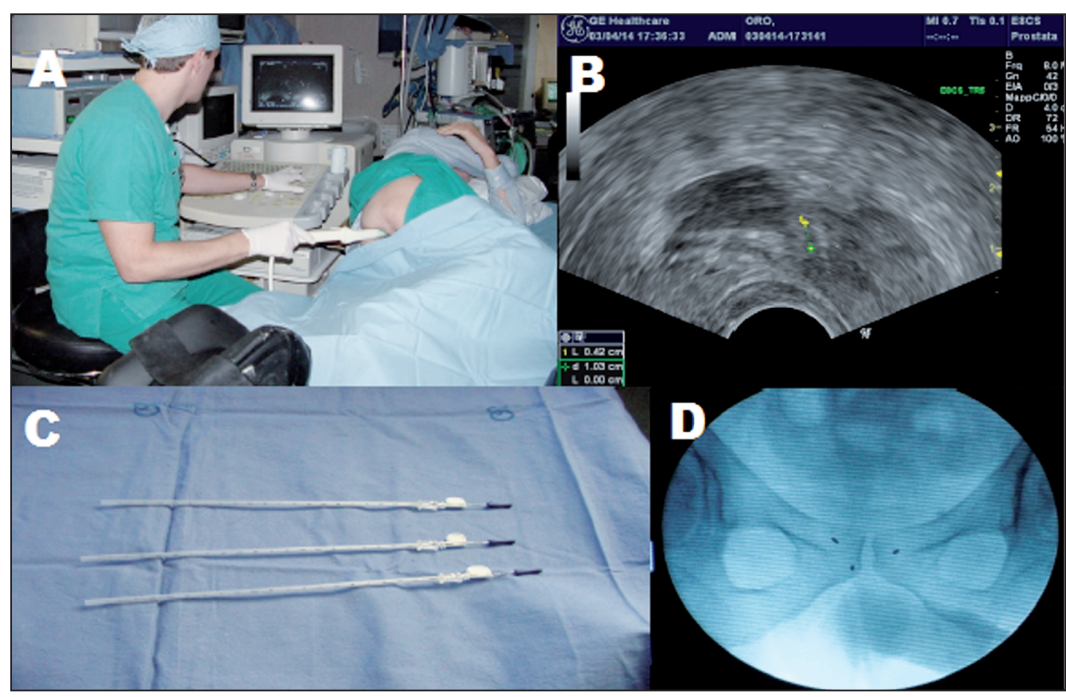

the marker. Each marker was released in the right position according to anatomy of the gland and radiation oncologist's preference with a triangulation scheme (avoiding the gold markers overlapping on the 3 orthogonal planes). The correct gold markers position was verified by fluoroscopy in the first 50 cases (Figure 1).

\section{RESULTS}

All procedure were performed successfully under local anesthesia without any severe complication. Mean time was 20 minutes. Any marker displacement in the urethra, rectum or infection was recorded. The optimal triangulation scheme (avoiding the gold markers overlapping on the 3 orthogonal planes) was obtained in $95 \%$ of cases. In $5 \%$ of cases the spatial distribution on 3 different planes was difficult in very small prostate (volume $<22 \mathrm{cc}$ ) or in prostate with small later-lateral diameter (< $4 \mathrm{~cm}$ on axial plane). To avoid markers displacement, we keep away from large cyst $(>1 \mathrm{~cm})$ or close to the urethra lumen in patients who underwent previous surgery. Hyperechoic tissue areas mixed with parenchymal calcifications were avoided in order to improve ultrasound control and visibility. The end-fire probe equipped with biopsy track option viable on the monitor allows correct placement in all cases minimizing operator variability. Gold markers in most of case, but sometimes carbonium were used. Material does not change the technique. Patients with obstructive lower urinary tract symptoms before the procedure were treated with medical therapy. Both therapies alpha-blockers and 5-alfa reductase inhibitors were allowed during and after IGRT treatment. In particular, we added dutasteride or finasteride to treat urinary obstruction and clinical benign prostate enlargement if any antiandrogen or androgen block was used to cancer control.

Early complications: no cases of severe early complications are reported (bleeding, infections, abscess or markers migration). The commonest new symptom following the procedure was urinary frequency affecting $10 \%$ of patients. Hematuria, minor rectal bleeding, dysuria and haematospermia affected $5-10 \%$ of patients, all cases were self-limiting and of grade 1-2. Mean pain score was 2 (range 1-5) during the procedure (according VAS scale range from 0 to 10) (Table 1).

IGRT implication and late side effects: The PTV resulted thinner than standard ones for all the patients with a mean reduction of each diameter of 1 $\mathrm{mm}$ cranial, $1 \mathrm{~mm}$ caudal, and $3.5 \mathrm{~mm}$ anterior, $3 \mathrm{~mm}$ posterior, $2 \mathrm{~mm}$ left and $2.5 \mathrm{~mm}$ right. These characteristics result an overall reduction of the PTV of 37\% (range 23-59\%) (Figure 2).

The late toxicity was reported for $57 / 78$ patients with at least 12 months follow up: at a median follow-up of 34 months (range 12-84) we recorded 8/57 (14\%) Grade 1, 5/57 (8\%) Grade 
Table 1.

Our results compared to other studies in literature.

\begin{tabular}{|c|c|c|c|c|c|c|c|c|c|c|}
\hline \multirow[t]{2}{*}{ Study } & \multirow[t]{2}{*}{ No. pts } & \multirow{2}{*}{$\begin{array}{l}\text { Size gold marker } \\
\qquad(\mathrm{mm})\end{array}$} & \multirow[t]{2}{*}{ No. markers } & \multirow[t]{2}{*}{ Technique } & \multirow{2}{*}{$\begin{array}{l}\text { Technical } \\
\text { success }\end{array}$} & \multirow{2}{*}{$\begin{array}{c}\text { Markers } \\
\text { migration }\end{array}$} & \multicolumn{4}{|c|}{ Early complication (\%) } \\
\hline & & & & & & & G1 & G2 & G3 & G4 \\
\hline Deipolyi (7) & 111 & NR & 3 & Transrectal & $98 \%$ & NR & 0 & 0 & 0.9 & 0.9 \\
\hline Moman (8) & 914 & $5 \times 1$ & 3 & $\begin{array}{l}\text { Transrectal } \\
\text { Transperineal }\end{array}$ & NR & $0.18 \%$ & NR & NR & 2.0 & 0 \\
\hline Linden (4) & 98 & $5 \times 1.1$ & 3 & Transrectal & $100 \%$ & $0 \%$ & 0 & 0 & 0 & 0 \\
\hline Kably (9) & 75 & $3 \times 1.2$ & 4 & Transrectal & $99 \%$ & $0.3 \%$ & 6.6 & 2.6 & 0 & 1.3 \\
\hline Current study & 78 & $3 \times 0.9$ & 3 & Transrectal & $100 \%$ & $0 \%$ & 5.5 & 2.1 & 0 & 0 \\
\hline
\end{tabular}

Figure 2.

A: standard CTV-PTV margin of $10 \mathrm{~mm}$ (red line).

B: CTV-PTV (blue line) margin reduction with gold fiducial markers inserted.

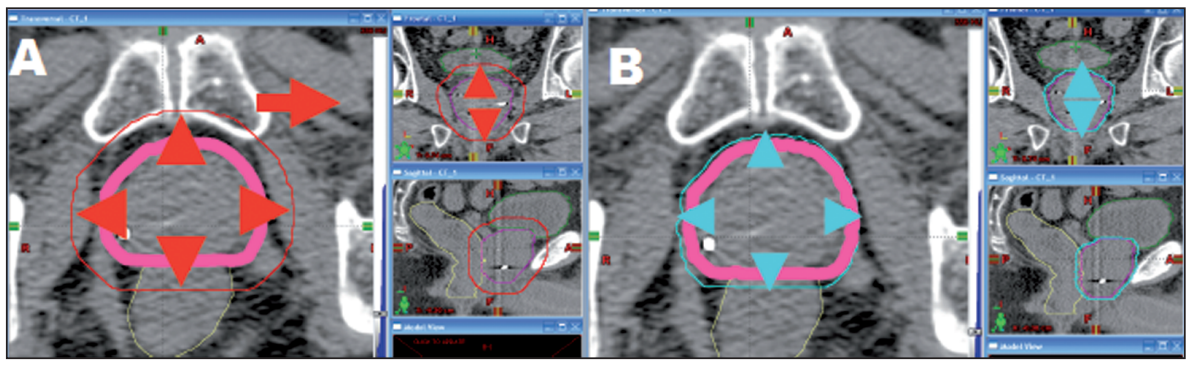

Table 2.

PTV reduction and late toxicity.

\begin{tabular}{|l|c|ccc|ccc|}
\hline \multirow{2}{*}{ IGRT with fiducial markers } & PTV reduction & \multicolumn{3}{|c|}{ Bladder toxicity } & \multicolumn{3}{|c|}{ Rectum toxicity } \\
& & G1 & G2 & G3 & G1 & G2 & G3 \\
\hline Current study & $37 \%(23-59)$ & $14 \%$ & $1.7 \%$ & $3.4 \%$ & $14 \%$ & $8 \%$ & $0 \%$ \\
\hline
\end{tabular}

be familiar with and skilled at the procedure.

Fiducial marker placement has been described using various techniques, including a transperineal route with endorectal US guidance, transrectally with an endorectal probe and transrectally through an endoscope; the number of fiducial markers placed ranges from three to five in most studies, with no evidence suggesting a superior number or configuration (4-6). In our experience we used three gold markers placed under TRUS guidance with a triangulation scheme that means trying to avoid the markers overlapping on the 3 orthogonal planes according to the radiation oncologists' preference: the 3 gold markers are usually

2 of late rectal toxicity; 8/57 (14\%) Grade 1, 1/57 (1.7\%) Grade 2, 2/57 (3.4\%) Grade 3 of late bladder toxicity according to LENT SOMA SCORE (Table 2).

\section{Discussion}

Differences in bladder and rectal filling as well as respiratory movements are known barriers to delivering increased radiation doses to the prostate using IGRT: fiducial markers provide a tool for reliable and accurate position verification before IGRT is delivered, allowing higher dose delivery to the target, while minimizing toxicities to surrounding normal tissue as reported in literature. With gold markers, for daily localization of the prostate, the margins around the gland can be reduced: several feasibility studies have shown the reliability of fiducial markers for prostate position verification during radiotherapy (1-3). So fiducial markers are being increasingly used by radiation oncologists to meet the dual objectives of delivering a higher dose to the PCA while sparing normal tissues so urologists are increasingly required to assist them during the TRUS-guided implantation: we believe that urologists should therefore released in the lateral mid left gland, right apex and base with some exceptions due to particular prostatic anatomy or gland's volume.

Based on our experience, the transrectal biplane probe is not useful to obtain the correct spatial distribution, since probe movement is limited in the rectum due to lenght and needle track visibility is limited compared with endfire probe.

With our end-fire TRUS guided technique described in this article we reported a very high technical success rate (all cases) and low early complications (0\% G3 and 2\% G2) similar to other experiences reported in literature: Deipolyi et al. (7) reported 98\% success rate of their technique using 3 fiducial markers in 111 consecutive localized PCA patients with only 0,9\% G3 early complication; Linden et al. (4) reported 100\% success rate of their technique using 1-3 fiducial markers in 98 consecutive localized PCA patients with no severe early toxicity. Moman et al. (8) reported the main experience in this field: 914 PCA patients, 3 fiducial markers inserted with a TRUS guided technique, 2/914 cases of severe early toxicity and 5/914 cases of markers migration; Kably et al. (9) reported 75 consecutive localized PCA patients with 
99\% of success rate and only $1.3 \%$ severe early complication with $0.3 \%$ markers migration. Other experiences are reported in literature by Gill et al. (10), Igdem et al. (11) and Langenhuijsen et al. (12) with similar results. There are few data quantifying the degree of spared healthy tissue with IGRT even in radiation oncology literature. Langenhuijsen et al. (13) reported a CTV-PTV margin reduction of $3 \mathrm{~mm}$ circumferentially, because of the use of gold markers, led to a mean PTV reduction of $27 \%$; in our experience the mean reduction of each diameter of PTV was $1 \mathrm{~mm}$ cranial, $1 \mathrm{~mm}$ caudal, 3.5 $\mathrm{mm}$ anterior, $3 \mathrm{~mm}$ posterior, $2 \mathrm{~mm}$ left and $2.5 \mathrm{~mm}$ right resulting a mean overall PTV reduction of $37 \%$. As expected, we reported a very low rate of late toxicity (3.4\% bladder G3 and 8\% rectal G2 side effects). Comparing the toxicity profiles between different studies is difficult, because the radiation techniques, doses, and treatment margins are different.

Limitations of the study include the relatively small patient population, it is not a prospective randomized study comparing IGRT with and without gold fiducial markers implantation, the follow-up is too short (mean 12 months) to clarify the favorable impact of fiducial on IGRT's late toxicity profile.

\section{Conclusions}

We report our technique of TRUS-guided fiducial gold markers implantation with its early and late complications in a group of PCA patients: it's a safe and well-tolerated procedure and it results helpful to reduce CTVPTV margin in all cases. As expected the late toxicity to surrounding normal tissues (bladder, rectum) resulted very low so the effects on clinical practice seem significant for both radiation oncologists and urologists who are involved in high-precision IGRT.

\section{REFERENCES}

1. Dehnad H, Nederveen AJ, van der Heide UA, et al. Clinical feasibility study for the use of implanted gold seeds in the prostate as reliable positioning markers during megavoltage irradiation. Radiother Oncol. 2003; 67:295-302.

2. Shirato H, Harada T, Harabayashi T, et al. Feasibility of insertion/implantation of 2.0-mm-diameter gold internal fiducial markers for precise setup and real-time tumor tracking in radiotherapy. Int J Radiat Oncol Biol Phys. 2003; 56:240-7.

3. Cardinali M, Galosi AB, Fenu F, et al. Adaptative radiotherapy with gold markers to reduce radiotherapy-relateed toxitcy in low risk prostate cancer patients. Anticancer Research. 2011; 31:1889-90.

4. Linden RA, Weiner PR, Gomella LG, et al. Technique of outpatient placement of intraprostatic fiducial markers before external beam radiotherapy. Urology. 2009; 73:881-6.

5. Shinohara K, Roach M 3rd. Technique for implantation of fiducial markers in the prostate. Urology 2008; 71:196-200

6. Henry AM, Wilkinson C, Wylie JP, et al. Transperineal implantation of radio-opaque treatment verification markers into the prostate: an assessment of procedure related morbidity, patient acceptability and accuracy. Radiother Oncol. 2004; 73:57-59.

7. Deipolyi AR, Mueller P, Efstathiou J, et al. Transrectal ultrasound-guided prostate fiducial marker placementfor prostate local- ization during external beam radiotherapy: a safe and reliable procedure. J Vasc Interv Radiol. 2013; 24:S98-S99.

8. Moman MR, van der Heide UA, Kotte AN, et al. Long-term experience with transrectal and transperineal implantations of fiducial gold markers in the prostate for position verification in external beam radiotherapy; feasibility, toxicity and quality of life. Radiother Oncol. 2010; 96:38-42.

9. Kably I, Bordegaray M, Shah K, et al. Single-Center Experience in Prostate Fiducial Marker Placement: Technique and Midterm Follow-up. J Vasc Interv Radiol 2014; 25:1125-1132.

10. Gill S, Li J, Thomas J, et al. Patient-reported complications from fiducial marker implantation for prostate image-guided radiotherapy. Br J Radiol. 2012; 85:1011-7.

11. Igdem S, Akpinar H, Alço $G$, et al. Implantation of fiducial markers for image guidance in prostate radiotherapy: patientreportedtoxicity. Br J Radiol. 2009; 82:941-5.

12. Langenhuijsen JF, van Lin EN, Kiemeney LA, et al. Ultrasoundguided transrectal implantation of gold markers for prostate localization during external beam radiotherapy: complication rate and risk factors. Int J Radiat Oncol Biol Phys. 2007; 69:671-6.

13. Langenhuijsen JF, Smeenk RJ, Louwe RJ, et al. Reduction of treatment volume and radiation doses to surrounding tissues withcntraprostatic gold markers in prostate cancer radiotherapy. Clin Genitourin Cancer. 2011; 9:109-113.

\section{Correspondence}

Vito Lacetera, MD (Corresponding Author) vlacetera@gmail.com

Giulia Sbrollini, MD, Resident of Urology giuliasbrollini@libero.it

Flavia Tombolini, MD, Resident of Urology

flavia.tombolini@virgilio.it

Alessandro Conti, MD, Urologist

alessandro.conti@hotmail.com

Giovanni Muzzonigro, MD, Urologist g.muzzonigro@univpm.it

Clinica Urologica, Azienda Ospedaliero-Universitaria Ospedali Riuniti Via Conca 71 - 61100 Torrette di Ancona, Italy

\section{Massimo Cardinali MD, Radiotherapist} massimo.cardinali@ospedaliriuniti.marche.it

Giovanna Mantello, MD,Radiotherapist giovanna.mantello@ospedaliriuniti.marche.it

Francesco Fenu, MD, Radiotherapist

francesco.fenu@ospedaliriuniti.marche.it

SOD Radioterapia Azienda Ospedaliero-Universitaria Ospedali Riuniti, Università Politecnica delle Marche, Ancona, Italy

Stefania Maggi, Medical Physic

stefania.maggi@ospedaliriuniti.marche.it

SOD Fisica Sanitaria, Azienda Ospedaliero-Universitaria Ospedali Riuniti, Università Politecnica delle Marche, Ancona, Italy

Andrea Galosi, MD, Urologist

galosiab@yahoo.it

UOC di Urologia, Ospedale "Augusto Murri", ASUR Marche, Fermo, Italy 